\title{
Expanded Programme on Immunization in Bangladesh: A Success Story
}

\author{
PROBIR KUMAR SARKAR ${ }^{1}$, NITAL KUMAR SARKER ${ }^{2}$, SHARMIM DOULAH $^{3}$, TAJUL ISLAM A. BARI ${ }^{4}$
}

\begin{abstract}
Infections are responsible for the majority of loss of life in children and vaccination has made enormous contribution to public health, including the eradication of one dreaded disease, small pox, and elimination of poliomyelitis from all but a handful of countries. Globally, immunization currently averts an estimated 2 to 3 million deaths every year. In Bangladesh it has prevented an estimated 2 million deaths from 19872000, and continues to prevent approximately 200,000 deaths each year. WHO introduced EPI (Expanded Programme on Immunization) in 1977 at Alma-Ata, the capital of Kazakhastan for the underdeveloped countries. Subsequently Bangladesh has launched EPI programme. Recently $A D$ (Auto Disable) syringes and vaccination against Hepatitis B, H. Influenzae, Measles-Rubella (MR) and Pneumococcus has been introduced in vaccination programme. In Bangladesh, immunization coverage of DPT/PENTA3 was only $16 \%$ in 1988 increased significantly to $69 \%$ in $1990,81 \%$ in $2000,90 \%$ in 2011 and $92 \%$ in 2013 which was higher than global coverage of $84 \%$ in 2013 and comparable to first world countries. In Pakistan fully immunization coverage in 2010 was only $50 \% .9$ Indian national full immunization coverage was $56 \%$ in 1990, 59\% in 2000, 74\% in 2010 and $74 \%$ in 2012 whereas in our country it was $52 \%$ in $1991,53 \%$ in $2000,79 \%$ in $2010,80 \%$ in 2011 and $81 \%$ in 2013 which signifies our excellent success for prevention of communicable diseases in successive years. So, EPI in Bangladesh has been recognized for its sustained high coverage and great contribution to the reduction of childhood morbidity and mortality and it received two 'GAVI best performance award' in 2009 and 2012.
\end{abstract}

Key Words: EPI Programme, Immunization

\section{History}

The WHO officially launched a global immunization programme in May 1974, known as 'Expanded Programme on Immunization' (EPI) to protect all children of the World against six vaccine preventable diseases, namely tuberculosis, polio, diphtheria, whooping cough, tetanus and measles by the year 2000. In Bangladesh EPI was formally launched on 7th April 1979 as a pilot project in eight thanas, is now the most successful programme of Bangladesh Government. At the outset in 1979, EPI started with six conventional vaccines- BCG, DPT, OPV, TT and

1. Resident Physician (Assistant Professor), Dhaka Shishu Hospital.

2. Assistant Professor, Paediatric Nephrology, Dhaka Shishu Hospital.

3. Surveillance Medical Officer, World Health Organization.

4. Program Manager, EPI \& Surveillance, DGHS.

Correspondence: Dr. Probir Kumar Sarkar. Email: tultulprobir@ yahoo.com
Measles. As vaccination centres were few and were located mainly in health care facilities in urban areas, the EPI coverage remained less than $2 \%$ by $1984 .{ }^{1}$ In 1985, the People's Republic of Bangladesh committed to the Global Universal Child Immunization Initiative $(\mathrm{UCI})$, and began a phase-wise process of EPI intensification from 1985-1990. During this time period, EPI was intensified throughout 476 Upazila, 92 major Municipalities and 6 City Corporations. Finally EPI was made available to all target groups (infants and pregnant mothers) by 1990 .

EPI intensification consisted of establishing the cold chain system from EPI Headquarter to District and Upazila level and capacity to maintain cold chain down to the vaccination points in rural and urban areas, procuring and managing logistics needs for about 134,000 EPI outreach sites, and providing basic EPI training for thousands of mid-level managers, supervisors and field workers in the public and private sectors. ${ }^{2,} 3$ 
In the year 1993 Government of Bangladesh endorsed TT5 dose schedule for women of child bearing age initially 15 to 45 years age and later extend to 15 to 49 years age.

Polio eradication and Maternal \& Neonatal tetanus elimination activities initiated in 1995. As a part of this Acute Flaccid Paralysis (AFP), Measles and Neonatal tetanus surveillance initiated in 1997.

During the last few years, based on the data on disease burden, new vaccine for selected emerging diseases such as Hepatitis B (2003) and Hib Disease (2009) have been incorporated into the EPI schedule with GAVI (Global Alliance for Vaccines and Immunization) support in pentavalent formulation $(\mathrm{DPT}+\mathrm{HepB}+\mathrm{Hib})$ that has added advantage of reducing shots for vaccination and minimize concern of mothers for use of injectable vaccine.

In view of enhancing the injection safety $A D$ (Auto Disable) syringes were introduced in to the programme from 2004.Then MR and MCV2 (Measles Containing Vaccine second dose) introduced in September 2012 and finally Pneumococcal Conjugate Vaccine (PCV) and Injectable Polio Vaccine (IPV) in March $2015 .^{2}$

Since 1995 two days are set every year as National Immunization Days (NIDs) usually during the dry winter months to facilitate wide participation, were conducted with very high (around 90\%) coverage in view of eradicating Polio and measles catch up campaign in 2005.

Surveillance activity in EPI for Vaccine Preventable Diseases (VPDs) started in 1995 with AFP surveillance for Polio eradication. After that more diseases and issues have been incorporated for surveillance in EPINT, Measles and AEFI. As a result of many interventions like Maternal and Neonatal Tetanus (MNT) elimination campaign, Measles catch up campaign, NID for Polio eradication a commendable success has been achieved in immunization program. The country has achieved the threshold of NT validation from 2008. Since 2003 Bangladesh has strengthened measles surveillance. No laboratory confirmed measles outbreak has yet been detected since conduction of measles catch up campaign in 20052006 and measles follow-up campaign in 2010 . So measles surveillance has been shifted from outbreak investigation to case based surveillance at facility level. The country is again maintaining polio free status from 22 November 2006 after experiencing importation in early part of that year. ${ }^{2,3,4}$ Finally, Bangladesh along with 10 other countries of WHO South-East-Asia Region was certified polio free on March 27, 2014 by an independent commission under the WHO certification process. ${ }^{5}$

\section{Introduction}

Vaccination has made enormous contribution to public health, including the eradication of one dreaded disease, small pox, and elimination of poliomyelitis from all but a handful of countries. ${ }^{6}$ Bangladesh has made significant progress in recent times in many of its social development indicators particularly in health. This country has made important gains in providing primary health care since the Alma Ata Declaration in 1977. All health indicators show steady gains and the health status of the population has improved. Immunization has been one of Bangladesh's greatest public health success stories. ${ }^{2}$ As a result of outstanding performance in improving the child immunization status, Bangladesh achieved Global Alliance for Vaccines and Immunization (GAVI)Alliance Award in 2009 and 2012, which is given as a recognition to achieving the Millennium Development Goals (MDG), particularly in reducing child mortality. ${ }^{3}$

\section{Epidemiology}

It is estimated that between two and three million child deaths are averted annually through vaccination against diphtheria, tetanus, pertussis and measles and many more future deaths averted in older groups (e. g. 600,000 future deaths prevented annually through hepatitis $B$ vaccination). $5,7,8$ However, vaccine preventable diseases are still responsible for about $25 \%$ of the 10 million deaths occurring annually among children under five years of age. ${ }^{5}$ Infectious diseases responsible for 8.3 million of total 12.5 million deaths of under five children globally in the year 1990.6 In Bangladesh it has prevented an estimated 2 million deaths from 1987-2000, and continues to prevent approximately 200,000 deaths each year. ${ }^{2}$ One out of five infants worldwide, nearly $20 \%$ of children is still unprotected against vaccine-preventable illness and over $70 \%$ of these unreached children live in ten countries: Afganistan, Chad, The Democratic Republic of Congo, Ethiopia, India, Indonesia, Nigeria, Pakistan, The Philippines and South Africa. Notwithstanding in 2013, an estimated 21.8 million infants worldwide were not reached with routine immunization services, of whom nearly half live in 3 countries, viz. India, Pakistan and Nigeria. ${ }^{7,8}$ 
The comprehensive multi-year plan (cmyp) of the national immunization program of bangladesh 2011-2016:

The cMYP provides a framework to plan activities to achieve important objectives of the National Immunization Program, as contained in the National Health Policy. This Plan sets out the medium-term (2011-2016) strategic goals of the immunization program, the related objectives, indicators, milestones, key activities and the associated costing and funding plan.

Bangladesh cMYP for the immunization program is based on the Global Immunization Vision and Strategy (GIVS) - ratified by the World Health Assembly in May 2005.

National Objectives, Strategies and Key activities 2011-2016:

Objective 1. Improve immunization coverage among children under one and child bearing age women, namely

1) At least $90 \%$ fully immunization coverage among under one children at national level and $85 \%$ full immunization coverage at district level.

2) TT5 coverage among women of childbearing age reached at least $80 \%$ at national level and $75 \%$ at each district level.

\section{Objective 2. Maintain polio free status}

By effectively implementing Acute Flaccid Paralysis (AFP) Surveillance, conducting supplementary OPV vaccination (NIDs/SNIDSs), mop-up OPV campaigns, and maintaining high routine OPV coverage Bangladesh managed to maintain the polio free status since 2006. In the presence of ongoing polio outbreaks in neighbouring India and presence of few districts with relatively low OPV coverage, Bangladesh need to put extra effort to intensify the above strategies in coming years to maintain the polio free status in the country.

Objective 3. Maintain maternal and neonatal tetanus elimination status

Achieved elimination status of MNT in Bangladesh (2008) is a major public health success, but significant challenge remains to maintain this status especially due to the fact that $80 \%$ of deliveries still taking place outside the institutions. Other than that TT complete coverage (TT5) among child bearing age females remain around $53 \%$. In view of maintaining this MNNT elimination status, following broad Strategies are incorporated into the CMYP

- Maintain high coverage of TT5 among childbearing age women

- Maintain high TT protection at birth

- Intensify current NT surveillance

- Introduce Td among school age children

Objective 4. Achieve national level $95 \%$ measles coverage and reaching measles elimination status by 2016

In 2009, EPI programme has managed to reach national level measles- 1 coverage among infants over $90 \%$, still 11 out of $64(17 \%)$ districts reported measles coverage less than $80 \%$.

Over the last few years Bangladesh EPI programme managed to control the morbidity and mortality associated with measles up to a significant level by maintaining high coverage of measles- 1 among infants, immunizing 35 million children from age 9 months to 10 years during the measles catch up programme in 2006 and immunizing all children aged nine months to sixty months during measles follow-up campaign in year 2010. To achieve the measles elimination status by 2016 , Bangladesh EPI programme need to intensify the measles control activities in coming years. For that, following broad Strategies are incorporated into the CMYP

- Maintain high MCV1 coverage among infants with special emphasis to the low coverage districts

- Intensity measles surveillance

- Introduction of Measles 2nd dose to the EPI schedule

\section{Objective 5. Prevention of diseases protected} by new and underused vaccines

Bangladesh Government and EPI programme is planning to introduce Pneumococcal vaccine, Rota vaccine, Birth dose of hepatitis $B$ vaccine, second dose of measles vaccine and $\mathrm{Td}$ vaccine in to the national EPI programme in coming years with GAVI support. To achieve this objective following broad Strategies are incorporated into the CMYP

- Strengthen coordination with development partners, local NGOs/CBOs, institutions 
BANGLADESH J CHILD HEALTH 2015; VOL 39 (2) : 96 Expanded Programme on Immunization in Bangladesh: A Success Story

- Establishment of surveillance system for diseases covered by new antigens.

- Introduction of new vaccines according to the planned timeline

- Ensure the future financial sustainability

Objective 6. Ensure safe injection practices and waste disposal

For the last few years Bangladesh EPI programme exclusively use AD syringes for all EPI vaccinations. When we consider the number of antigens administered, reported number of AEFI seems to be far less than the expected. Though under HPSP and later HNPSP, government of Bangladesh identified medical waste management as a priority area, still there is no proper EPI waste management mechanism in place. Majority of the Upazila use pit burning method to dispose medical wastes. To address this important area in future, following broad Strategies are incorporated into the cMYP. ${ }^{2}$

- AEFI surveillance system strengthened

- Implementation on national plan on sharp and waste management for EPI waste

- Strengthen AEFI surveillance system

- Ensure injection safety
Table-I

Immunization Coverage Percentage in Bangladesh as per EPI Coverage Evaluation Survey.

\begin{tabular}{lcccc}
\hline Year & BCG & $\begin{array}{c}\text { DPT3/ } \\
\text { Penta3 }\end{array}$ & Measles & $\begin{array}{c}\text { Fully } \\
\text { Vaccinated }\end{array}$ \\
\hline 2000 & 95 & 81 & 61 & 53 \\
2001 & 94 & 85 & 64 & 52 \\
2002 & 95 & 86 & 65 & 56 \\
2003 & 95 & 87 & 69 & 63 \\
2005 & 95 & 77 & 71 & 64 \\
2006 & 98 & 84 & 78 & 71 \\
2007 & 98 & 87 & 81 & 75 \\
2009 & 99 & 86 & 83 & 75 \\
2010 & 99 & 89 & 85 & 79 \\
2011 & 99 & 90 & 86 & 80 \\
2013 & 99 & 92 & 86 & 81 \\
\hline
\end{tabular}

Source: Coverage Evaluation Survey 2000-2002, 2003, 2005, 2006, 2007, 2009, 2010, 2011 and 2013.

Table-III

Global Immunization Coverage

\begin{tabular}{ccccc}
\hline Year & DTP3/Penta3 & Hepatitis B & Hib & Measles \\
\hline 2013 & $84 \%$ & $81 \%$ & $52 \%$ & $84 \%$ \\
2012 & $83 \%$ & $79 \%$ & $45 \%$ & $84 \%$ \\
2011 & $83 \%$ & $75 \%$ & $43 \%$ & $84 \%$ \\
\hline
\end{tabular}

Source: Global Immunization Data WHO/UNICEF

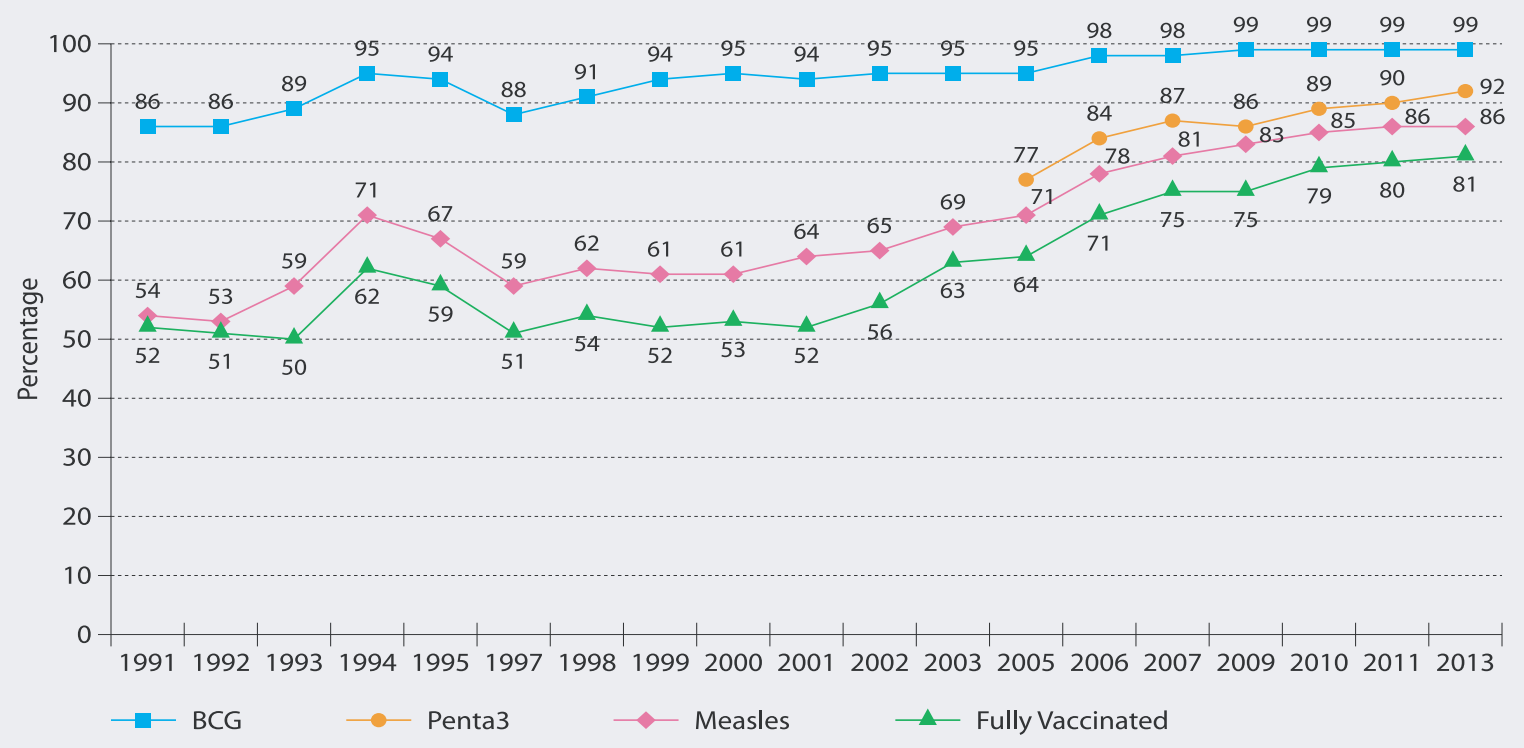

Source : Coverage Evaluation Survey 1991-1995, 1997-2002, 2003, 2005, 2006, 2007, 2009, 2010, 2011 and 2013

Fig.-1: Annual trend in national valid vaccination coverage by age 12 months among 12-23 months old children from 1991 to 2013 
Table-III

Routine Immunization Coverage Estimates, 2008-2012, SEAR Countries

\begin{tabular}{lllllllllllllllllllllll}
\hline Country & \multicolumn{1}{c}{ BCG } & \multicolumn{1}{c}{ DPT3/PENTA3 } & \multicolumn{1}{c}{ OPV3 } \\
\hline Yr 2008-12 & 08 & 09 & 10 & 11 & 12 & 08 & 09 & 10 & 11 & 12 & 08 & 09 & 10 & 11 & 12 & 08 & 09 & 10 & 11 & 12 \\
Bangladesh & 90 & 94 & 94 & 95 & 95 & 95 & 96 & 95 & 96 & 96 & 95 & 96 & 95 & 96 & 96 & 96 & 98 & 94 & 96 & 96 \\
India & 87 & 87 & 87 & 87 & 87 & 72 & 72 & 72 & 72 & 72 & 70 & 70 & 70 & 70 & 70 & 74 & 74 & 74 & 74 & 74 \\
Sri Lanka & 99 & 99 & 99 & 99 & 99 & 98 & 97 & 99 & 99 & 99 & 98 & 97 & 99 & 99 & 99 & 98 & 97 & 99 & 99 & 99 \\
Nepal & 87 & 94 & 94 & 97 & 96 & 82 & 89 & 82 & 92 & 90 & 82 & 93 & 83 & 92 & 90 & 79 & 90 & 86 & 88 & 86 \\
Maldives & 99 & 99 & 97 & 98 & 99 & 98 & 98 & 96 & 96 & 99 & 98 & 98 & 97 & 96 & 99 & 97 & 98 & 97 & 96 & 98 \\
DPR Korea & 97 & 98 & 98 & 98 & 98 & 92 & 93 & 93 & 94 & 96 & 98 & 98 & 99 & 99 & 99 & 98 & 98 & 99 & 99 & 99 \\
Myanmar & 88 & 93 & 93 & 93 & 87 & 85 & 90 & 90 & 86 & 85 & 85 & 90 & 90 & 90 & 87 & 82 & 87 & 88 & 88 & 84 \\
Indonesia & 80 & 78 & 80 & 80 & 81 & 65 & 62 & 62 & 62 & 64 & 72 & 67 & 66 & 65 & 69 & 76 & 74 & 75 & 74 & 80 \\
Thailand & 99 & 99 & 99 & 99 & 99 & 99 & 99 & 99 & 99 & 99 & 99 & 99 & 99 & 99 & 99 & 98 & 98 & 98 & 98 & 98 \\
Bhutan & 99 & 94 & 96 & 95 & 95 & 96 & 93 & 91 & 95 & 97 & 96 & 93 & 92 & 95 & 97 & 99 & 94 & 95 & 95 & 95 \\
\hline
\end{tabular}

\section{Discussion}

Emerging consciousness to control infectious diseases worldwide proved significant result. In Bangladesh, EPI is implemented through various outreach centers and clinic-based activities. The service delivery mechanism for providing EPI services in rural areas relies on a system of 64 Districts, 482 Upazilas, 4498 Unions, 13,494 Wards, and 108,000 sub-blocks within the wards. Each sub-block has an EPI outreach site where routine EPI services are provided monthly for catchments of approximately 1000 populations. ${ }^{2}$ Motivation and education on immunization are provided during the household visits by the health workers, who are known as Health Assistants (HA), and family planning fieldworkers, called Family Welfare Assistants (FWA). The vaccination services are available at the outreach sites like EPI sites, satellite clinics, static clinics and community clinics. ${ }^{1,2}$ The Global Immunization Vision and Strategy (GIVS) was developed by WHO and UNICEF as a framework for strengthening national immunization programmes and protect as many as people as possible against more diseases by expanding the reach of immunization. ${ }^{6}$

Immunization coverage in India in four different provinces were $75 \%, 58 \%, 57 \%$ and $56 \%$ in 2007 and full immunization coverage in rural India was $39 \%$ in 2010 whereas it was $75 \%$ in Bangladesh in 2007. In Pakistan fully immunization coverage in 2010 was only
$50 \%{ }^{9,10}$ Indian national full immunization coverage was $56 \%$ in $1990,59 \%$ in $2000,74 \%$ in 2010 and $74 \%$ in 2012 whereas it was $52 \%$ in $1991,53 \%$ in $2000,79 \%$ in 2010 and $81 \%$ in 2011 which signifies our excellent success for prevention of communicable diseases in successive years. Global DPT/PENTA3 immunization coverage was $83 \%$ in 2011 \& 2012 and $84 \%$ in 2013 . Moreover we proved our excellence to cover DPT3/PENTA3 compared first world countries which was only $16 \%$ in 1988 increased significantly to $69 \%$ in $1990,81 \%$ in 2000 and $90 \%$ in $2011.11-15$

The trend of immunization coverage- a key measure of immunization system performance shows that the immunization program has strong capacity to reach children with BCG (99\%), DPT-3/Penta-3 (89\%), Polio-3 (89\%) and Measles (86\%). Though only $(81 \%)$ of children one year of age are fully immunized with all doses of vaccines which they are supposed to receive during the first year of life. The Coverage Evaluation Survey (CES)-2013 found fully vaccinated $81 \%$ children which is $1 \%$ higher than the previous year, but $9 \%$ below the national target of $90 \%$ to be achieved by 2016. Though Bangladesh is considerably better positioned than India, Pakistan and other SEAR countries, it is not enough which clearly indicated by the data of Sri Lanka, Maldives and Thailand. So there is a lot of room for improvement and this in made possible by implementation of the EPI. 


\section{Conclusion}

The Expanded Programme on Immunization (EPI) is one of the most successful public health interventions in Bangladesh, and has contributed significantly to reducing mortality and morbidity from vaccinepreventable diseases. The service delivery mechanism of EPI throughout the country has been used as the role model and a platform to deliver other interventions. The latest report shows that the coverage of the fully vaccinated children who had taken the doses as per schedule before celebrating their first birth day was 81 percent in 2013. Though the rate is still much lower than the government's target of 90 percent by 2016, the Expanded Programme of Immunization (EPI) that oversees child immunization finds it to be "a success". The coverage has been consistently increasing by one percentage point for the last six years. Bangladesh is much appreciated for its high vaccine coverage in routine immunization and it received two 'GAVI best performance award' within the past six years.

\section{References}

1. Islam MD, Alam HSK, Islam MR. EPI programme: An excellent success for prevention of communicable diseases in Bangladesh. DS (CHILD) HJ 2010; 26:113-18.

2. Comprehensive Multi-Year Plan of the National Immunization Program of Bangladesh 2011-16, Expanded Programme on Immunization, DGHS, Mohakhali, Dhaka 1212.

3. Bangladesh EPI Coverage Evaluation Survey 2013, Expanded Programme on Immunization, DGHS, Mohakhali, Dhaka 1212. Annual Trend in National Valid vaccination Coverage by age 12 months among 12-23 months old children from 1991 to 2013. 2013:33.

4. National Measles Elimination, Rubella Control and Polio Eradication Programme, Planning and Implementation Guide 2013, Expanded Programme on Immunization, DGHS, Mohakhali, Dhaka 1212.
5. Challenges in global immunization and the global immunization vision and strategy 2006-20015. http://www.who.int/wer/2006/wer8119.pdf, accessed on 20/03/2015.

6. P Duclos, J M O Bele, K G Dobo, T Cherisn. Global Immunization: status, progress, challenges and future. BMC International Health and Human Rights 2009; 9(1):S2.

7. Global Immunization Data 2012, WHO \& UNICEF. http://www.who.int/immunization_ monitoring/data/en/, accessed on 02/02/2014.

8. Global Immunization Data 2013, WHO \& UNICEF. http://www.who.int/immunization monitoring/data/en/, accessed on 02/02/2014.

9. Expanded Program on Immunization (EPI): A Bangladeshi Success Story. https:// drfahimahmad.wordpress.com/2012/12/23/ expanded-program-on-immunization-epi-abangladeshi-success-story/epi-success.

10. EPI in Pakistan, Fafheen Zafar Butt Saba Ahmed. http://www.scribd.com/doc/22576862/ EPI-in-Pakistan accessed on 29/09/2010.

11. World Health Organization, EPI Fact Sheet, SEAR, 2012. http://www.searo.who.int/topics/ immunization, accessed on 15/01/2014.

12. World Health Organization, EPI Fact Sheet, India, 2013. http://www.searo.who.int/topics/ immunization, accessed on 15/01/2014.

13. World Health Organization, EPI Fact Sheet, Bangladesh, 2013. http://www.searo.who.int/ topics/immunization, accessed on 15/01/2014.

14. World Health Organization, EPI Fact Sheet, Sri Lanka, 2013. http://www.searo.who.int/topics/ immunization, accessed on 18/02/2014.

15. World Health Organization, EPI Fact Sheet, Maldives, 2013. http://www.searo.who.int/topics/ immunization, accessed on 18/02/2014. 